Bull. Korean Math. Soc. 48 (2011), No. 6, pp. 1195-1205

http://dx.doi.org/10.4134/BKMS.2011.48.6.1195

\title{
GENERALIZED WEIGHTED COMPOSITION OPERATORS FROM AREA NEVANLINNA SPACES TO WEIGHTED-TYPE SPACES
}

\author{
YANG WEIFENG AND YAN WEIREN
}

\begin{abstract}
Let $H(\mathbb{D})$ denote the class of all analytic functions on the open unit disk $\mathbb{D}$ of the complex plane $\mathbb{C}$. Let $n$ be a nonnegative integer, $\varphi$ be an analytic self-map of $\mathbb{D}$ and $u \in H(\mathbb{D})$. The generalized weighted composition operator is defined by

$$
D_{\varphi, u}^{n} f=u f^{(n)} \circ \varphi, f \in H(\mathbb{D}) .
$$
\end{abstract}

The boundedness and compactness of the generalized weighted composition operator from area Nevanlinna spaces to weighted-type spaces and little weighted-type spaces are characterized in this paper.

\section{Introduction}

Let $\mu$ be a positive continuous function on $[0,1)$. We say that $\mu$ is normal if there exist $\delta \in[0,1)$ and two positive numbers $a$ and $b$ with $0<a<b$, such that (see [21])

$$
\begin{aligned}
& \frac{\mu(r)}{(1-r)^{a}} \text { is decreasing on }[\delta, 1), \lim _{r \rightarrow 1} \frac{\mu(r)}{(1-r)^{a}}=0 ; \\
& \frac{\mu(r)}{(1-r)^{b}} \text { is increasing on }[\delta, 1), \lim _{r \rightarrow 1} \frac{\mu(r)}{(1-r)^{b}}=\infty .
\end{aligned}
$$

A non-negative function $\|\cdot\|$ on a vector space $X$ (over the real or complex field $K$ ) is called an F-norm if the following properties are satisfied:

(i) $\|x\|=0 \Leftrightarrow x=0$;

(ii) $\|\lambda x\| \leq\|x\|$ for all $\lambda \in K$ with $|\lambda| \leq 1$;

(iii) $\|x+y\| \leq\|x\|+\|y\|$ for all $x, y \in X$;

(iv) if $\lambda_{m} \rightarrow 0$ and $\lambda_{m} \in K$, then $\left\|\lambda_{m} x\right\| \rightarrow 0$.

An F-norm $\|\cdot\|$ induces a transitive invariant distance $d$ by $d(x, y)=\|x-y\|$ for all $x, y \in X$. A vector space $X$ with an $\mathrm{F}$-norm $\|\cdot\|$ is said to be an $\mathrm{F}^{*}$-space. A complete $\mathrm{F}^{*}$-space is called an $\mathrm{F}$-space.

Received June 30, 2010; Revised January 13, 2011.

2010 Mathematics Subject Classification. Primary 47B38; Secondary 30H05.

Key words and phrases. generalized weighted composition operator, area Nevanlinna space, weighted-type space. 
Let $\mathbb{D}$ be the open unit disk in the complex plane $\mathbb{C}$. We denote by $H(\mathbb{D})$ the class of all holomorphic functions on $\mathbb{D}$. Let $p \in[1, \infty), \alpha>-1$. An $f \in H(\mathbb{D})$ is said to belong to the area Nevanlinna space $\mathcal{N}_{\alpha}^{p}=\mathcal{N}_{\alpha}^{p}(\mathbb{D})$, if

$$
\|f\|_{\mathcal{N}_{\alpha}^{p}}^{p}=\int_{\mathbb{D}}[\log (1+|f(z)|)]^{p} d A_{\alpha}(z)<\infty
$$

where $d A_{\alpha}(z)=\left(1-|z|^{2}\right)^{\alpha} d A(z)$. It is easy to see that

$$
\|f+g\|_{\mathcal{N}_{\alpha}^{p}} \leq\|f\|_{\mathcal{N}_{\alpha}^{p}}+\|g\|_{\mathcal{N}_{\alpha}^{p}}
$$

for all $f, g \in \mathcal{N}_{\alpha}^{p}$. Consequently, $\mathcal{N}_{\alpha}^{p}$ becomes a metric space.

Also, we have by subharmonicity

$$
\log (1+|f(z)|) \leq C \frac{\|f\|_{\mathcal{N}_{\alpha}^{p}}}{\left(1-|z|^{2}\right)^{(2+\alpha) / p}}, f \in \mathcal{N}_{\alpha}^{p},
$$

where $C$ depends only on $p$ and $\alpha$ (see, e.g. [2]).

From (1) and (2), it is easy to verify that $\|\cdot\|_{\mathcal{N}_{\alpha}^{p}}$ is an F-norm of the space $\mathcal{N}_{\alpha}^{p}$. Moreover, from (2), it follows that if $f_{m} \rightarrow f$ in $\mathcal{N}_{\alpha}^{p}$, then $f_{m} \rightarrow f$ locally uniformly. Here, locally uniform convergence means uniform convergence on every compact subset of $\mathbb{D}$. Therefore, under the F-norm, $\mathcal{N}_{\alpha}^{p}$ becomes an F-space, i.e., a translation-invariant complete metric space.

In this paper, a subset $A$ of $\mathcal{N}_{\alpha}^{p}$ is called bounded if there exists a positive number $r$ such that $A \subset\left\{f \in \mathcal{N}_{\alpha}^{p}:\|f\|_{\mathcal{N}_{\alpha}^{p}}<r\right\}$. Given a Banach space $X$, we say that a linear map $T: \mathcal{N}_{\alpha}^{p} \rightarrow X$ is bounded if $T(A) \subset X$ is bounded for every bounded subset $A$ of $\mathcal{N}_{\alpha}^{p}$. We say that $T$ is compact if $T(A) \subset X$ is relatively compact for every bounded subset $A \subset \mathcal{N}_{\alpha}^{p}$.

Suppose $\mu$ is a normal function on $[0,1)$. An analytic function $f$ on $\mathbb{D}$ is said to belong to the weighted-type space $\mathcal{H}_{\mu}^{\infty}=\mathcal{H}_{\mu}^{\infty}(\mathbb{D})$, if

$$
\|f\|_{\mathcal{H}_{\mu}^{\infty}}=\sup _{z \in \mathbb{D}} \mu(|z|)|f(z)|<\infty .
$$

The little weighted-type space $\mathcal{H}_{\mu, 0}^{\infty}=\mathcal{H}_{\mu, 0}^{\infty}(\mathbb{D})$ is a subspace of $\mathcal{H}_{\mu}^{\infty}$ consisting of all $f \in H(\mathbb{D})$ such that

$$
\lim _{|z| \rightarrow 1^{-}} \mu(|z|)|f(z)|=0 .
$$

See [1] for more information on $\mathcal{H}_{\mu}^{\infty}$.

Let $n$ be a nonnegative integer, $\varphi$ be an analytic self-map of $\mathbb{D}$ and $u \in H(\mathbb{D})$. The generalized weighted composition operator $D_{\varphi, u}^{n}$ is defined by

$$
D_{\varphi, u}^{n} f=u f^{(n)} \circ \varphi, f \in H(\mathbb{D}),
$$

where $f^{(0)}=f$. The generalized weighted composition operator $D_{\varphi, u}^{n}$ can be regarded as a product of composition operator $C_{\varphi}$, multiplication operator $M_{u}$ and the $n$-th differentiation operator $D^{n}$. The generalized weighted composition operator $D_{\varphi, u}^{n}$ was introduced in [38], and studied in [25,31, 38, 39, 41, $43,29,30]$. 
It is interesting to provide a function theoretic characterization of the conditions under which the generalized weighted composition operator $D_{\varphi, u}^{n}$ becomes a bounded or compact operator on various spaces of analytic functions. The books $[4,36]$ contain plenty of information on this topic for $D_{\varphi, u}^{n}$ in the case of $n=0$ and $u(z)=1$, i.e., for the composition operator $C_{\varphi}$.

In the case of $n=0, D_{\varphi, u}^{n}$ is the weighted composition operator $u C_{\varphi}$. Weighted composition operators between analytic function spaces have been studied in $[3,5,9,10,11,12,13,16,18,20,22,23,26,31,34,37,40,42]$ (see also related references therein). Xiao studied the compact composition operator on the area Nevanlinna space in [32], and characterized the boundedness and compactness of the composition operator from the area Nevanlinna space to the Bloch space in [33]. Zhu studied the weighted composition operator from the area Nevanlinna space to the Bloch space in [42].

The case $n=1$ and $u(z)=\varphi^{\prime}(z)$, that is $D_{\varphi, u}^{n}=D C_{\varphi}$, was studied in $[6,8,27,35]$. The case $n=1$ and $u(z)=1$, that is $D_{\varphi, u}^{n}=C_{\varphi} D$, was studied in $[6,19,35,28,14,27,24,15]$.

This paper focuses on the boundedness and compactness of the generalized weighted composition operator from the area Nevanlinna space to the weighted-type space and the little weighted-type space. Some sufficient and necessary conditions for the generalized weighted composition operator $D_{\varphi, u}^{n}$ to be bounded or compact are given.

Throughout this paper $C$ denotes a positive constant which may be differ from one occurrence to another.

\section{Main results and proofs}

In this section, we will give our main results and proofs. In order to prove our main results, we need some auxiliary results which are incorporated in the following lemmas.

Lemma 1. Let $n$ be a nonnegative integer, $1 \leq p<\infty$ and $\alpha>-1$. Then there exists some $C$ such that for each $f \in \mathcal{N}_{\alpha}^{p}$ and $z \in \mathbb{D}$,

$$
\left|f^{(n)}(z)\right| \leq \frac{1}{\left(1-|z|^{2}\right)^{n}} \exp \left[\frac{C\|f\|_{\mathcal{N}_{\alpha}^{p}}}{\left(1-|z|^{2}\right)^{\frac{2+\alpha}{p}}}\right] .
$$

Proof. For $z \in \mathbb{D}$ and $\xi \in \partial \mathbb{D}$, we have

$$
1-\left|z+\frac{1-|z|}{2} \xi\right|^{2} \geq 1-\frac{(1+|z|)^{2}}{4} \geq \frac{1-|z|^{2}}{4} .
$$

From this, the Cauchy's integral formula for derivatives and (2), we have

$$
\begin{aligned}
\left|f^{(n)}(z)\right| & =\left|\frac{n !}{2 \pi i} \int_{\partial \mathbb{D}} \frac{f(\xi)}{(\xi-z)^{n+1}} d \xi\right| \\
& \leq \frac{n ! 2^{n}}{2 \pi(1-|z|)^{n}} \int_{\partial \mathbb{D}}\left|f\left(z+\frac{1-|z|}{2} \xi\right)\right||d \xi|
\end{aligned}
$$




$$
\begin{aligned}
& \leq \frac{1}{2 \pi\left(1-|z|^{2}\right)^{n}} \int_{\partial \mathbb{D}}\left(1+n ! 4^{n}\left|f\left(z+\frac{1-|z|}{2} \xi\right)\right|\right)|d \xi| \\
& \leq \frac{1}{2 \pi\left(1-|z|^{2}\right)^{n}} \int_{\partial \mathbb{D}} \exp \left[n ! 4^{n} \log \left(1+\left|f\left(z+\frac{1-|z|}{2} \xi\right)\right|\right)\right]|d \xi| \\
& \leq \frac{1}{2 \pi\left(1-|z|^{2}\right)^{n}} \int_{\partial \mathbb{D}} \exp \left[\frac{C\|f\|_{\mathcal{N}_{\alpha}^{p}}}{\left(1-\left|z+\frac{1-|z|}{2} \xi\right|^{2}\right)^{\frac{2+\alpha}{p}}}\right]|d \xi| \\
& \leq \frac{1}{2 \pi\left(1-|z|^{2}\right)^{n}} \int_{\partial \mathbb{D}} \exp \left[\frac{C\|f\|_{\mathcal{N}_{\alpha}^{p}}}{\left(\frac{1-|z|^{2}}{4}\right)^{\frac{2+\alpha}{p}}}\right]|d \xi| \\
& =\frac{1}{\left(1-|z|^{2}\right)^{n}} \exp \left[\frac{C\|f\|_{\mathcal{N}_{\alpha}^{p}}^{N^{2}}}{\left(1-|z|^{2}\right)^{\frac{2+\alpha}{p}}}\right]
\end{aligned}
$$

from which the result follows.

The proof of the following lemma is similar to the proof of Lemma 1 in [17], so we omit it here.

Lemma 2. Suppose $\mu$ is a normal function on $[0,1)$. A closed set $K$ in $\mathcal{H}_{\mu, 0}^{\infty}$ is compact if and only if it is bounded and satisfies

$$
\lim _{|z| \rightarrow 1} \sup _{f \in K} \mu(|z|)|f(z)|=0 .
$$

The following criterion for compactness follows from arguments similar to those in Proposition 2.3 of [7].

Lemma 3. Suppose that $\varphi$ is an analytic self-map of $\mathbb{D}, u \in H(\mathbb{D}), 1 \leq p<\infty$, $\alpha>-1$ and $\mu$ is a normal function on $[0,1)$. Then the operator $D_{\varphi, u}^{n}: \mathcal{N}_{\alpha}^{p} \rightarrow$ $\mathcal{H}_{\mu}^{\infty}$ is compact if and only if for each sequence $\left\{f_{k}\right\}_{k \in \mathbb{N}}$ which is bounded in $\mathcal{N}_{\alpha}^{p}$ and converges to zero locally uniformly on $\mathbb{D}$, we have $\left\|D_{\varphi, u}^{n} f_{k}\right\|_{\mathcal{H}_{\mu}^{\infty}} \rightarrow 0$ as $k \rightarrow \infty$.

Now, we are ready to state and prove the main results of this paper.

Theorem 1. Suppose that $\varphi$ is an analytic self-map of $\mathbb{D}, u \in H(\mathbb{D}), 1 \leq p<$ $\infty, \alpha>-1$ and $\mu$ is a normal function on $[0,1)$. Then for each nonnegative integer $n, D_{\varphi, u}^{n}: \mathcal{N}_{\alpha}^{p} \rightarrow \mathcal{H}_{\mu}^{\infty}$ is bounded if and only if for all $c>0$,

$$
M(c)=: \sup _{z \in \mathbb{D}} \frac{\mu(|z|)|u(z)|}{\left(1-|\varphi(z)|^{2}\right)^{n}} \exp \left[\frac{c}{\left(1-|\varphi(z)|^{2}\right)^{\frac{2+\alpha}{p}}}\right]<\infty .
$$

Proof. Suppose that $D_{\varphi, u}^{n}: \mathcal{N}_{\alpha}^{p} \rightarrow \mathcal{H}_{\mu}^{\infty}$ is bounded. Let $f(z)=\frac{z^{n}}{n !} \in \mathcal{N}_{\alpha}^{p}$. Then we have

$$
\|u\|_{\mathcal{H}_{\mu}^{\infty}}=\left\|D_{\varphi, u}^{n} f\right\|_{\mathcal{H}_{\mu}^{\infty}} \leq\left\|D_{\varphi, u}^{n}\right\|_{\mathcal{N}_{\alpha}^{p} \rightarrow \mathcal{H}_{\mu}^{\infty}}\|f\|_{\mathcal{N}_{\alpha}^{p}}<\infty .
$$

Hence $u(z) \in \mathcal{H}_{\mu}^{\infty}$. 
For any $c>0$ and $z \in \mathbb{D}$, take

$$
f_{z}(\omega)=\exp \left\{c\left[\frac{1-|\varphi(z)|^{2}}{(1-\overline{\varphi(z)} \omega)^{2}}\right]^{\frac{2+\alpha}{p}}\right\} .
$$

By using the inequality $\left|e^{u}\right| \leq e^{|u|}, u \in \mathbb{C}$, we have

$$
\int_{\mathbb{D}}\left[\log \left(1+\left|f_{z}(\omega)\right|\right)\right]^{p} d A_{\alpha}(\omega) \leq \int_{\mathbb{D}} c^{p}\left|\frac{1-|\varphi(z)|^{2}}{(1-\overline{\varphi(z)} \omega)^{2}}\right|^{2+\alpha} d A_{\alpha}(\omega)<\infty .
$$

Hence $f_{z} \in \mathcal{N}_{\alpha}^{p}$ for all $z \in \mathbb{D}$.

On the other hand,

$$
\begin{aligned}
f_{z}^{(n)}(\omega)= & {\left[\frac{c_{0} A^{n} \overline{(\varphi(z))^{n}}}{(1-\overline{\varphi(z)} w)^{\frac{2 n(2+\alpha)}{p}}+n}+\frac{c_{1} \overline{(\varphi(z))^{n}} A^{n-1}}{(1-\overline{\varphi(z)} w)^{\frac{2(n-1)(2+\alpha)}{p}}+n}+\cdots\right.} \\
& \left.+\frac{c_{n-1} \overline{(\varphi(z))^{n}} A}{(1-\overline{\varphi(z)} w)^{\frac{2(2+\alpha)}{p}+n}}\right] \exp \left\{c\left[\frac{1-|\varphi(z)|^{2}}{(1-\overline{\varphi(z)} \omega)^{2}}\right]^{\frac{2+\alpha}{p}}\right\}, \omega \in \mathbb{D},
\end{aligned}
$$

where $A=\left(1-|\varphi(z)|^{2}\right)^{\frac{2+\alpha}{p}}$ and $c_{0}, c_{1}, \ldots, c_{n-1}$ are positive numbers depending only on $c, \alpha, n$ and $p$, for example, $c_{0}=\left(\frac{2 c(2+\alpha)}{p}\right)^{n}$. Then

$$
\begin{aligned}
& \left\|D_{\varphi, u}^{n} f_{z}\right\|_{\mathcal{H}_{\mu}^{\infty}} \\
& =\sup _{\omega \in \mathbb{D}} \mu(|\omega|)\left|u(\omega) f_{z}^{(n)}(\varphi(\omega))\right| \\
& \geq \mu(|z|)\left|u(z) f_{z}^{(n)}(\varphi(z))\right| \\
& =\mu(|z|)|u(z)| \exp \left\{c\left[\frac{1-|\varphi(z)|^{2}}{\left(1-|\varphi(z)|^{2}\right)^{2}}\right]^{\frac{2+\alpha}{p}}\right\} \times \mid \frac{c_{0} A^{n} \overline{(\varphi(z))^{n}}}{\left(1-|\varphi(z)|^{2}\right)^{\frac{2 n(2+\alpha)}{p}+n}} \\
& +\frac{c_{1} \overline{(\varphi(z))^{n}} A^{n-1}}{\left(1-|\varphi(z)|^{2}\right)^{\frac{2(n-1)(2+\alpha)}{p}+n}}+\cdots+\frac{c_{n-1} \overline{(\varphi(z))^{n}} A}{\left(1-|\varphi(z)|^{2}\right)^{\frac{2(2+\alpha)}{p}+n}} \mid \\
& =\mu(|z|)|u(z)||\varphi(z)|^{n} \exp \left[\frac{c}{\left(1-|\varphi(z)|^{2}\right)^{\frac{2+\alpha}{p}}}\right] \times \mid \frac{c_{0}}{\left(1-|\varphi(z)|^{2}\right)^{\frac{n(2+\alpha)}{p}+n}} \\
& +\frac{c_{1}}{\left(1-|\varphi(z)|^{2}\right)^{\frac{(n-1)(2+\alpha)}{p}+n}}+\cdots+\frac{c_{n-1}}{\left(1-|\varphi(z)|^{2}\right)^{\frac{2+\alpha}{p}+n}} \mid \\
& \geq \frac{c_{0} \mu(|z|)|u(z)||\varphi(z)|^{n}}{\left(1-|\varphi(z)|^{2}\right)^{\frac{n(2+\alpha)}{p}+n}} \exp \left[\frac{c}{\left(1-|\varphi(z)|^{2}\right)^{\frac{2+\alpha}{p}}}\right],
\end{aligned}
$$

which implies that

(6) $\frac{\mu(|z|)|u(z)|}{\left(1-|\varphi(z)|^{2}\right)^{n}} \exp \left[\frac{c}{\left(1-|\varphi(z)|^{2}\right)^{\frac{2+\alpha}{p}}}\right] \leq \frac{\left\|D_{\varphi, u}^{n} f_{z}\right\|_{\mathcal{H}_{\mu}^{\infty}}\left(1-|\varphi(z)|^{2}\right)^{\frac{n(2+\alpha)}{p}}}{c_{0}|\varphi(z)|^{n}}$. 
Applying (6), it is easy to see that

$$
\sup _{\left\{z \in \mathbb{D}:|\varphi(z)|>\frac{1}{2}\right\}} \frac{\mu(|z|)|u(z)|}{\left(1-|\varphi(z)|^{2}\right)^{n}} \exp \left[\frac{c}{\left(1-|\varphi(z)|^{2}\right)^{\frac{2+\alpha}{p}}}\right]<\infty .
$$

Moreover, from $u(z) \in \mathcal{H}_{\mu}^{\infty}$, we have

$$
\begin{aligned}
& \sup _{\left\{z \in \mathbb{D}:|\varphi(z)| \leq \frac{1}{2}\right\}} \frac{\mu(|z|)|u(z)|}{\left(1-|\varphi(z)|^{2}\right)^{n}} \exp \left[\frac{c}{\left(1-|\varphi(z)|^{2}\right)^{\frac{2+\alpha}{p}}}\right] \\
\leq & \left(\frac{4}{3}\right)^{n} \exp \left[c\left(\frac{4}{3}\right)^{\frac{2+\alpha}{p}}\right] \sup _{z \in \mathbb{D}} \mu(|z|)|u(z)|<\infty .
\end{aligned}
$$

From (7) and (8), it follows that condition (5) holds.

Conversely, suppose (5) holds. Let $S$ be a bounded subset in $\mathcal{N}_{\alpha}^{p}$. Then there exists a positive number $K$ such that $\|f\|_{\mathcal{N}_{\alpha}^{p}} \leq K$ for all $f \in S$. By Lemma 1 and (3), we have

$$
\begin{aligned}
\left\|D_{\varphi, u}^{n} f\right\|_{\mathcal{H}_{\mu}^{\infty}} & =\sup _{z \in \mathbb{D}} \mu(|z|)\left|\left(D_{\varphi, u}^{n} f\right)(z)\right| \\
& =\sup _{z \in \mathbb{D}} \mu(|z|)|u(z)|\left|f^{(n)}(\varphi(z))\right| \\
& \leq \sup _{z \in \mathbb{D}} \frac{\mu(|z|)|u(z)|}{\left(1-|\varphi(z)|^{2}\right)^{n}} \exp \left[\frac{C\|f\|_{\mathcal{N}_{\alpha}^{p}}}{\left(1-|\varphi(z)|^{2}\right)^{\frac{2+\alpha}{p}}}\right] \\
& =M(C K)<\infty
\end{aligned}
$$

for all $f \in S$. This implies $D_{\varphi, u}^{n}(S)$ is a bounded subset of $\mathcal{H}_{\mu}^{\infty}$, and then $D_{\varphi, u}^{n}: \mathcal{N}_{\alpha}^{p} \rightarrow \mathcal{H}_{\mu}^{\infty}$ is a bounded operator. The proof is completed.

Theorem 2. Suppose that $\varphi$ is an analytic self-map of $\mathbb{D}, u \in H(\mathbb{D}), 1 \leq p<$ $\infty, \alpha>-1$ and $\mu$ is a normal function on $[0,1)$. Then for each nonnegative integer $n, D_{\varphi, u}^{n}: \mathcal{N}_{\alpha}^{p} \rightarrow \mathcal{H}_{\mu}^{\infty}$ is compact if and only if $u(z) \in \mathcal{H}_{\mu}^{\infty}$ and for all $c>0$,

$$
\lim _{|\varphi(z)| \rightarrow 1} \frac{\mu(|z|)|u(z)|}{\left(1-|\varphi(z)|^{2}\right)^{n}} \exp \left[\frac{c}{\left(1-|\varphi(z)|^{2}\right)^{\frac{2+\alpha}{p}}}\right]=0
$$

Proof. Suppose that $D_{\varphi, u}^{n}: \mathcal{N}_{\alpha}^{p} \rightarrow \mathcal{H}_{\mu}^{\infty}$ is compact. Then it is clear that $D_{\varphi, u}^{n}: \mathcal{N}_{\alpha}^{p} \rightarrow \mathcal{H}_{\mu}^{\infty}$ is bounded. Now from the proof of Theorem 1, we can conclude $u(z) \in \mathcal{H}_{\mu}^{\infty}$

Let $\left\{z_{k}\right\}$ be a sequence such that $\left|\varphi\left(z_{k}\right)\right| \rightarrow 1$ as $k \rightarrow \infty$. For any $c>0$, set

$$
f_{k}(\omega)=\exp \left\{c\left[\frac{1-\left|\varphi\left(z_{k}\right)\right|^{2}}{\left(1-\overline{\varphi\left(z_{k}\right)} \omega\right)^{2}}\right]^{\frac{2+\alpha}{p}}\right\}-1 \text {. }
$$

It is easy to see that the sequence $\left\{f_{k}\right\}$ converges to zero local uniformly on any compact subsets of $\mathbb{D}$. Moreover, from the proof of Theorem 1 , we can see $\left\{f_{k}\right\}$ is a bounded sequence in $\mathcal{N}_{\alpha}^{p}$. 
Since $D_{\varphi, u}^{n}$ is compact, by Lemma 3 we have

$$
\left\|D_{\varphi, u}^{n} f_{k}\right\|_{\mathcal{H}_{\mu}^{\infty}}=0 .
$$

On the other hand, (6) implies

$$
\frac{\mu\left(\left|z_{k}\right|\right)\left|u\left(z_{k}\right)\right|}{\left(1-\left|\varphi\left(z_{k}\right)\right|^{2}\right)^{n}} \exp \left[\frac{c}{\left(1-\left|\varphi\left(z_{k}\right)\right|^{2}\right)^{\frac{2+\alpha}{p}}}\right] \leq \frac{\left\|D_{\varphi, u}^{n} f_{k}\right\|_{\mathcal{H}_{\mu}^{\infty}}\left(1-\left|\varphi\left(z_{k}\right)\right|^{2}\right)^{\frac{n(2+\alpha)}{p}}}{c_{0}\left|\varphi\left(z_{k}\right)\right|^{n}} .
$$

Taking $k \rightarrow \infty$ on both sides of above inequality, we obtain

$$
\lim _{k \rightarrow \infty} \frac{\mu\left(\left|z_{k}\right|\right)\left|u\left(z_{k}\right)\right|}{\left(1-\left|\varphi\left(z_{k}\right)\right|^{2}\right)^{n}} \exp \left[\frac{c}{\left(1-\left|\varphi\left(z_{k}\right)\right|^{2}\right)^{\frac{2+\alpha}{p}}}\right]=0
$$

which implies that (9) holds.

Conversely, suppose $u(z) \in \mathcal{H}_{\mu}^{\infty}$ and (9) holds. Then for every $c, \varepsilon>0$, there is a $\delta \in(0,1)$ such that

$$
\frac{\mu(|z|)|u(z)|}{\left(1-|\varphi(z)|^{2}\right)^{n}} \exp \left[\frac{c}{\left(1-|\varphi(z)|^{2}\right)^{\frac{2+\alpha}{p}}}\right]<\varepsilon,
$$

whenever $\delta<|\varphi(z)|<1$.

Now let $\left\{f_{k}\right\}$ be a sequence in $\mathcal{N}_{\alpha}^{p}$ such that $f_{k} \rightarrow 0$ local uniformly on $\mathbb{D}$ and $\|f\|_{\mathcal{N}_{\alpha}^{p}} \leq K$. Then $f_{k}^{(n)} \rightarrow 0$ local uniformly on $\mathbb{D}$. Therefore using (3) and (4), we have

$$
\begin{aligned}
\left\|D_{\varphi, u}^{n} f_{k}\right\|_{\mathcal{H}_{\mu}^{\infty}}= & \sup _{z \in \mathbb{D}} \mu(|z|)\left|\left(D_{\varphi, u}^{n} f_{k}\right)(z)\right| \\
= & \sup _{z \in \mathbb{D}} \mu(|z|)|u(z)|\left|f_{k}^{(n)}(\varphi(z))\right| \\
\leq & \sup _{\{z \in \mathbb{D}:|\varphi(z)| \leq \delta\}} \mu(|z|)|u(z)|\left|f_{k}^{(n)}(\varphi(z))\right| \\
& +\sup _{\{z \in \mathbb{D}: \delta<|\varphi(z)|<1\}} \mu(|z|)|u(z)|\left|f_{k}^{(n)}(\varphi(z))\right| \\
\leq & \|u\|_{\mathcal{H}_{\mu}^{\infty}} \sup _{\{w \in \mathbb{D}:|w| \leq \delta\}}\left|f_{k}^{(n)}(w)\right| \\
& +\sup _{\{z \in \mathbb{D}: \delta<|\varphi(z)|<1\}} \frac{\mu(|z|)|u(z)|}{\left(1-|\varphi(z)|^{2}\right)^{n}} \exp \left[\frac{C\left\|f_{k}\right\|_{\mathcal{N}_{\alpha}^{p}}}{\left(1-|\varphi(z)|^{2}\right)^{\frac{2+\alpha}{p}}}\right] \\
\leq & \|u\|_{\mathcal{H}_{\mu}^{\infty}} \sup _{\{w \in \mathbb{D}:|w| \leq \delta\}}\left|f_{k}^{(n)}(w)\right| \\
& +\sup _{\{z \in \mathbb{D}: \delta<|\varphi(z)|<1\}} \frac{\mu(|z|)|u(z)|}{\left(1-|\varphi(z)|^{2}\right)^{n}} \exp \left[\frac{C K}{\left(1-|\varphi(z)|^{2}\right)^{\frac{2+\alpha}{p}}}\right] \\
\leq & \|u\|_{\mathcal{H}_{\mu}^{\infty}} \sup _{\{w \in \mathbb{D}:|w| \leq \delta\}}\left|f_{k}^{(n)}(w)\right|+\varepsilon .
\end{aligned}
$$


Note that $u(z) \in \mathcal{H}_{\mu}^{\infty}$ and the positive number $\varepsilon$ is arbitrary. This yields

$$
\lim _{k \rightarrow \infty}\left\|D_{\varphi, u}^{n} f_{k}\right\|_{\mathcal{H}_{\mu}^{\infty}}=0 .
$$

It follows from Lemma 3 that the operator $D_{\varphi, u}^{n}: \mathcal{N}_{\alpha}^{p} \rightarrow \mathcal{H}_{\mu}^{\infty}$ is compact. The proof is completed.

From Theorems 1 and 2, we can obtain the following corollary.

Corollary 3. Suppose that $\varphi$ is an analytic self-map of $\mathbb{D}, u \in H(\mathbb{D}), 1 \leq p<$ $\infty, \alpha>-1$ and $\mu$ is a normal function on $[0,1)$. Then for each nonnegative integer $n$, the following are equivalent:

(i) $D_{\varphi, u}^{n}: \mathcal{N}_{\alpha}^{p} \rightarrow \mathcal{H}_{\mu}^{\infty}$ is bounded;

(ii) $D_{\varphi, u}^{n}: \mathcal{N}_{\alpha}^{p} \rightarrow \mathcal{H}_{\mu}^{\infty}$ is compact;

(iii) $u(z) \in \mathcal{H}_{\mu}^{\infty}$ and for all $c>0$ condition (9) hold.

Proof. From the proof of Theorem 2, we can get (iii) $\Rightarrow($ ii), and (ii) $\Rightarrow$ (i) is obvious.

(i) $\Rightarrow$ (iii). Suppose that $D_{\varphi, u}^{n}: \mathcal{N}_{\alpha}^{p} \rightarrow \mathcal{H}_{\mu}^{\infty}$ is bounded. By Theorem 1, $u(z) \in \mathcal{H}_{\mu}^{\infty}$. Moreover, for all $\delta, c>0$, we have $M(c)<\infty$ and $M(c+\delta)<\infty$. Using the equation

$$
\lim _{|\varphi(z)| \rightarrow 1} \exp \left[\frac{\delta}{\left(1-|\varphi(z)|^{2}\right)^{\frac{2+\alpha}{p}}}\right]=\infty,
$$

we conclude that the condition (9) holds for all $c>0$. The proof is completed.

Theorem 4. Suppose that $\varphi$ is an analytic self-map of $\mathbb{D}, u \in H(\mathbb{D}), 1 \leq p<$ $\infty, \alpha>-1$ and $\mu$ is a normal function on $[0,1)$. Then for each nonnegative integer $n$, the following are equivalent:

(i) $D_{\varphi, u}^{n}: \mathcal{N}_{\alpha}^{p} \rightarrow \mathcal{H}_{\mu, 0}^{\infty}$ is bounded;

(ii) $D_{\varphi, u}^{n}: \mathcal{N}_{\alpha}^{p} \rightarrow \mathcal{H}_{\mu, 0}^{\infty}$ is compact;

(iii) $u(z) \in \mathcal{H}_{\mu, 0}^{\infty}$ and for all $c>0$,

$$
\lim _{|z| \rightarrow 1} \frac{\mu(|z|)|u(z)|}{\left(1-|\varphi(z)|^{2}\right)^{n}} \exp \left[\frac{c}{\left(1-|\varphi(z)|^{2}\right)^{\frac{2+\alpha}{p}}}\right]=0 .
$$

Proof. (ii) $\Rightarrow$ (i). It is obvious.

(i) $\Rightarrow$ (iii). Suppose that $D_{\varphi, u}^{n}: \mathcal{N}_{\alpha}^{p} \rightarrow \mathcal{H}_{\mu, 0}^{\infty}$ is bounded. For $f(z)=z^{n} \in \mathcal{N}_{\alpha}^{p}$, it follows that $u(z) \in \mathcal{H}_{\mu, 0}^{\infty}$. Since $D_{\varphi, u}^{n}: \mathcal{N}_{\alpha}^{p} \rightarrow \mathcal{H}_{\mu}^{\infty}$ is bounded, by Corollary 3 , we conclude that the condition (9) hold for all $c>0$. Thus, for each $c, \varepsilon>0$, there exists a number $t \in(0,1)$ such that

$$
\frac{\mu(|z|)|u(z)|}{\left(1-|\varphi(z)|^{2}\right)^{n}} \exp \left[\frac{c}{\left(1-|\varphi(z)|^{2}\right)^{\frac{2+\alpha}{p}}}\right]<\varepsilon,
$$

whenever $t<|\varphi(z)|<1$. 
Moreover, from $u(z) \in \mathcal{H}_{\mu, 0}^{\infty}$, we infer that there exists a number $r \in(0,1)$ such that for $r<|z|<1$,

$$
\mu(|z|)|u(z)|<\frac{\varepsilon}{\left(1-t^{2}\right)^{n}} \exp \left[\frac{-c}{\left(1-t^{2}\right)^{\frac{2+\alpha}{p}}}\right] .
$$

Therefore, if $r<|z|<1$ and $|\varphi(z)|>t$, then by (12) we have

$$
\frac{\mu(|z|)|u(z)|}{\left(1-|\varphi(z)|^{2}\right)^{n}} \exp \left[\frac{c}{\left(1-|\varphi(z)|^{2}\right)^{\frac{2+\alpha}{p}}}\right]<\varepsilon .
$$

If $r<|z|<1$ and $|\varphi(z)| \leq t$, then by (13) we have

$$
\frac{\mu(|z|)|u(z)|}{\left(1-|\varphi(z)|^{2}\right)^{n}} \exp \left[\frac{c}{\left(1-|\varphi(z)|^{2}\right)^{\frac{2+\alpha}{p}}}\right] \leq \frac{\mu(|z|)|u(z)|}{\left(1-t^{2}\right)^{n}} \exp \left[\frac{c}{\left(1-t^{2}\right)^{\frac{2+\alpha}{p}}}\right]<\varepsilon .
$$

In other words, for each $\varepsilon>0$, there exists an $r \in(0,1)$ such that for $r<|z|<$ 1 ,

$$
\frac{\mu(|z|)|u(z)|}{\left(1-|\varphi(z)|^{2}\right)^{n}} \exp \left[\frac{c}{\left(1-|\varphi(z)|^{2}\right)^{\frac{2+\alpha}{p}}}\right]<\varepsilon,
$$

which implies that (11) holds for all $c>0$.

(iii) $\Rightarrow$ (ii). Suppose that $u(z) \in \mathcal{H}_{\mu, 0}^{\infty}$ and (11) holds for all $c>0$. From Lemma $2, D_{\varphi, u}^{n}: \mathcal{N}_{\alpha}^{p} \rightarrow \mathcal{H}_{\mu, 0}^{\infty}$ is compact if and only if

(14) $\lim _{|z| \rightarrow 1} \sup _{f \in B_{\mathcal{N}_{\alpha}^{p}}} \mu(|z|)\left|\left(D_{\varphi, u}^{n} f\right)(z)\right|=\lim _{|z| \rightarrow 1} \sup _{f \in B_{\mathcal{N}_{\alpha}^{p}}} \mu(|z|)|u(z)|\left|f^{(n)}(\varphi(z))\right|=0$,

where $B_{\mathcal{N}_{\alpha}^{p}}=\left\{g \in \mathcal{N}_{\alpha}^{p}:\|g\|_{\mathcal{N}_{\alpha}^{p}} \leq 1\right\}$ is the unit ball in the space $\mathcal{N}_{\alpha}^{p}$.

On the other hand, by Lemma 1 , we have

$$
\mu(|z|)|u(z)|\left|f^{(n)}(\varphi(z))\right| \leq \frac{\mu(|z|)|u(z)|}{\left(1-|\varphi(z)|^{2}\right)^{n}} \exp \left[\frac{C\|f\|_{\mathcal{N}_{\alpha}^{p}}}{\left(1-|\varphi(z)|^{2}\right)^{\frac{2+\alpha}{p}}}\right] .
$$

Taking the supremum in (15) over the unit ball $B_{\mathcal{N}_{\alpha}^{p}}$, and letting $|z| \rightarrow 1$, from (11) we see that (14) hold and hence $D_{\varphi, u}^{n}: \mathcal{N}_{\alpha}^{p} \rightarrow \mathcal{H}_{\mu, 0}^{\infty}$ is compact. The proof is completed.

Acknowledgements. The authors wish to thank the referee for his/her useful comments.

\section{References}

[1] K. D. Bierstedt, J. Bonet, and J. Taskinen, Associated weights and spaces of holomorphic functions, Studia Math. 127 (1998), no. 2, 137-168.

[2] B. Choe, H. Koo, and W. Smith, Carleson measure for the area Nevalinna spaces and applications, J. Anal. Math. 104 (2008), 207-233.

[3] D. Clahane and S. Stević, Norm equivalence and composition operators between Bloch/Lipschitz spaces of the unit ball, J. Inequal. Appl. 2006 (2006), Article ID 61018.

[4] C. Cowen and B. MacCluer, Composition Operators on Spaces of Analytic Functions, Studies in Advanced Mathematics, CRC Press, Boca Raton, 1995.

[5] X. Fu and X. Zhu, Weighted composition operators on some weighted spaces in the unit ball, Abstr. Appl. Anal. 2008 (2008), Article ID 605807. 
[6] R. A. Hibschweiler and N. Portnoy, Composition followed by differentiation between Bergman and Hardy spaces, Rochy Mountain J. Math. 35 (2005), no. 3, 843-855.

[7] H. Jarchow and J. Xiao, Composition operators between Nevanlinna classes and Bergman spaces with weights, J. Operator Theory 46 (2001), no. 3, suppl., 605-618.

[8] S. Li and S. Stević, Composition followed by differentiation between Bloch type space, J. Comput. Anal. Appl. 9 (2007), no. 2, 195-205.

[9] — Weighted composition operators from $\alpha$-Bloch space to $H^{\infty}$ on the polydisk, Numer. Funct. Anal. Optim. 28 (2007), no. 7-8, 911-925.

[10] - Weighted composition operators from Bergman-type spaces into Bloch spaces, Proc. Indian Acad. Sci. Math. Sci. 117 (2007), no. 3, 371-385.

[11] Weighted composition operators from $H^{\infty}$ to the Bloch space on the polydisc, Abstr. Appl. Anal. 2007 (2007), Article ID 48478.

[12] Weighted composition operators between $H^{\infty}$ and $\alpha$-Bloch spaces in the unit ball, Taiwanese J. Math. 12 (2008), no. 7, 1625-1639.

[13] Weighted composition operators from Zygmund spaces into Bloch spaces, Appl. Math. Comput. 206 (2008), no. 2, 825-831.

[14] _ Composition followed by differentiation from mixed-norm spaces to $\alpha$-Bloch spaces, Matematicheskii Sbornik 199 (2008), no. 12, 117-128.

[15] Y. Liu and Y. Yu, Composition followed by differentiation between $H^{\infty}$ and Zygmund spaces, Complex Anal. Oper. Theory, (2010).

[16] Z. Lou, Composition operators on Bloch type spaces, Analysis (Munich) 23 (2003), no. 1, 81-95.

[17] K. Madigan and A. Matheson, Compact composition operators on the Bloch space, Trans. Amer. Math. Soc. 347 (1995), no. 7, 2679-2687.

[18] S. Ohno, Weighted composition operators between $H^{\infty}$ and the Bloch space, Taiwanese J. Math. 5 (2001), no. 3, 555-563.

[19] Products of composition and differentiation between Hardy spaces, Bull. Austral. Math. Soc. 73 (2006), no. 2, 235-243.

[20] S. Ohno, K. Stroethoff, and R. Zhao, Weighted composition operators between Bloch-type spaces, Rocky Mountain J. Math. 33 (2003), no. 1, 191-215.

[21] A. L. Shields and D. L. Williams, Bounded projections, duality, and multipliers in spaces of analytic functions, J. Reine Angew. Math. 299/300 (1978), 256-279.

[22] S. Stević, Weighted composition operators between mixed norm spaces and $H_{\alpha}^{\infty}$ spaces in the unit ball, J. Inequal. Appl. 2007 (2007), Article ID 28629.

[23] Weighted composition operators from weighted Bergman spaces to weighted-type spaces on the unit ball, Appl. Math. Comput. 212 (2009), no. 2, 499-504.

[24] _ Norm and essential norm of composition followed by differentiation from $\alpha$ Bloch spaces to $H_{\mu}^{\infty}$, Appl. Math. Comput. 207 (2009), no. 1, 225-229.

[25] W Wighted differentiation composition operators from mixed-norm spaces to weighted-type spaces, Appl. Math. Comput. 211 (2009), no. 1, 222-233.

[26] _ Norm of weighted composition operators from $\alpha$-Bloch spaces to weighted-type spaces, Appl. Math. Comput. 215 (2009), no. 2, 818-820.

[27] _ Products of composition and differentiation operators on the weighted Bergman space, Bull. Belg. Math. Soc. Simon Stevin 16 (2009), no. 4, 623-635.

[28] _ Composition followed by differentiation from $H^{\infty}$ and the Bloch space to nth weighted-type spaces on the unit disk, Appl. Math. Comput. 216 (2010), no. 12, 34503458.

[29] Weighted differentiation composition operators from $H^{\infty}$ and Bloch spaces to nth weighted-type spaces on the unit disk, Appl. Math. Comput. 216 (2010), no. 12, 3634-3641. 
[30] Weighted differentiation composition operators from the mixed-norm space to the nth weigthed-type space on the unit disk, Abstr. Appl. Anal. 2010 (2010), Article ID 246287.

[31] _ Weighted composition operators from the Bergman-Privalov-Type spaces to weighted-type spaces on the unit ball, Appl. Math. Comput. in press.

[32] J. Xiao, Compact composition operators on the area-Nevanlinna class, Exposition. Math. 17 (1999), no. 3, 255-264

[33] _ Composition operators: $\mathcal{N}_{\alpha}$ to the Bloch space to $\mathcal{Q}_{\beta}$, Studia Math. 139 (2000), no. 3, 245-260

[34] W. Yang, Weighted composition operators from Bloch-type spaces to weighted-type spaces, Ars Combin. 92 (2009), 415-423.

[35] _ Products of composition and differentiation operators from $\mathcal{Q}_{K}(p, q)$ spaces to Bloch-type spaces, Abstr. Appl. Anal. 2009 (2009), Art. ID 741920, 14 pp.

[36] K. Zhu, Operator Theory in Function Spaces, Marcel Dekker, Inc. Pure and Applied Mathematics 139, New York and Basel, 1990.

[37] X. Zhu, Weighted composition operators between $H^{\infty}$ and Bergman type spaces, Commun. Korean Math. Soc. 21 (2006), no. 4, 719-727.

[38] _ Products of differentiation, composition and multiplication operator from Bergman type spaces to Bers spaces, Integral Transforms Spec. Funct. 18 (2007), no. 3-4, 223-231.

[39] __ Generalized weighted composition operators from Bloch-type spaces to weighted Bergman spaces, Indian J. Math. 49 (2007), no. 2, 139-149.

[40] Weighted composition operators from $F(p, q, s)$ spaces to $H_{\mu}^{\infty}$ spaces, Abstr. Appl. Anal. 2009 (2009), Article ID 290978.

[41] _ Generalized weighted composition operators on weighted Bergman spaces, Numer. Funct. Anal. Optim. 30 (2009), no. 7-8, 881-893.

[42] Weighted composition operators from area Nevalinna spaces into Bloch spaces, Appl. Math. Comput. 215 (2010), no. 12, 4340-4346.

[43] Generalized weighted composition operators on Bloch-type spaces, Ars. Combin. to appear.

YANG WEIFENG

Department of Mathematics And Physics

Hunan Institute of EngineERING

411104, Xiangtan, Hunan, P. R. China

E-mail address: yangweifeng09@163.com

YAN WEIREN

Department of Mathematics and Physics

Hunan Institute of EngineEring

411104, Xiangtan, Hunan, P. R. China

E-mail address: ywr_2005@yahoo.com.cn 